\author{
Radmila Lazarević \\ Università del Montenegro
}

\title{
I SUFFISSI DETOPONIMICI NELLE LINGUE ITALIANA E SERBA
}

\begin{abstract}
Il contributo tratta la derivazione deonomastica dai toponimi mediante suffissi, e nella ricerca vengono messi a confronto i suffissi detoponimici italiani con quelli serbi. Adoperando l'approccio contrastivo, si analizzano le differenze e i tratti paralleli morfologici e semantici, partendo dalla classificazione morfologica dei suffissati. Viene dimostrato che i suffissati detoponimici rappresentano un campo onomastico che non comprende soltanto gli etnici (romano, belgradese, parigino), ma anche altri derivati piuttosto produttivi nelle lingue confrontate (italianista, arabismo, slavistico). Comunque, $\mathrm{i}$ derivati etnici rimangono la parte più numerosa $\mathrm{e}$, a loro volta, possono servire da base per ulteriori suffissi detoponimici (greco>grecizzare>grecizzazione). Il numero dei suffissi produttivi è limitato in entrambe le lingue, e più somiglianze si riscontrano nei suffissi "internazionali", di origine greca o latina.

Parole chiave: suffissi detoponimici, toponimi, suffissazione, etnici, lingua italiana, lingua serba.
\end{abstract}

\section{INTRODUZIONE}

I toponimi, o nomi propri di luoghi o di altri oggetti geografici (fiumi, laghi, monti ecc.) sono alla base di un intero campo onomastico dei cosiddetti detoponimici, termine usato per le parole deonomastiche (derivate dai nomi propri), che vengono formate di solito mediante la suffissazione (africano, milanese, parigino).

Questa ricerca mette a confronto i suffissi detoponimici italiani con quelli della lingua serba. Ci occuperemo dei detoponimici italiani e serbi soprattutto dal punto di vista della derivazione, limitandoci a quelli derivati mediante la suffissazione. Nella ricerca abbiamo consultato le rispettive grammatiche e gli studi sulla formazione delle parole ${ }^{1}$, facendo ricorso

*radmilal@ucg.ac.me

${ }^{1}$ Per l'italiano le opere: Grossmann e Rainer (2004), Dardano (2009), Crocco Galeas (1991) e il dizionario Neologismi: parole nuove dai giornali (2009) dell'Istituto Treccani. Per il serbo: Klajn (2003), Piper \& Klajn (2014), Klajn (2007), nonché Klajn e Šipka (2008). 
anche a dizionari cartacei e online. Il dizionario Neologismi: parole nuove dai giornali dell'Istituto Treccani si è dimostrato particolarmente utile nell'osservazione dei suffissi italiani più produttivi.

Sebbene oggi molti non siano tra i più produttivi nella formazione delle nuove parole, la maggioranza dei suffissi italiani che producono deonomastici sono detoponimici, e lo stesso vale anche per la maggioranza dei suffissi serbi, compresi quelli per formare il femminile. Semanticamente, la maggior parte dei suffissi detoponimici forma i cosiddetti etnici (per la definizione di https://www.treccani.it, nomi e aggettivi che determinano l'appartenenza a un popolo, a una nazione, a una regione o città), ma dato che la nostra ricerca riscontra pure alcuni suffissi produttivi con altri significati, parleremo di derivati detoponimici piuttosto che di quelli strettamente etnici. Inoltre, a differenza degli etnici, tra i suffissati detoponimici possono rientrare anche i verbi.

I derivati detoponimici e i suffissi che li producono possono essere classificati in più modi. Qui abbiamo optato per la classificazione morfologica: in suffissi nominali, aggettivali e verbali. Queste categorie verranno poi analizzate anche sotto l'aspetto semantico. Soltanto gli aggettivi e i nomi etnici, che sono i più numerosi e che nelle lingue confrontate non appartengono alle stesse categorie morfologiche, verranno analizzati in una sezione a parte.

\section{CLASSIFICAZIONE MORFOLOGICA}

Essendo derivati da nomi propri di luoghi e oggetti geografici, quasi tutti i detoponimici sono denominali, tranne alcuni deaggettivali e deverbali, derivati mediante l'accumulazione di suffissi che, comunque, hanno sempre la radice originaria nominale (ad es. it. America $>$ americano $>$ americanizzare $>$ americanizzazione, sr. Amerika > amerikanizovati / amerikanizirati $>$ amerikanizacija ${ }^{2}$.

Possono essere formati mediante suffissi nominali (it. -ista, -istica, -zione; sr. -ac, -ista, -anin), aggettivali (it. -(i)ano, -ese, -ico; sr. -ski, -istički) e verbali (it. -are, -eggiare, -izzare; sr. -izovati, -ovati, -čiti).

Nella lingua italiana, i cosiddetti etnici sono specifici, dato che possono appartenere sia ai nomi, se riferiti agli abitanti di un luogo, che agli aggettivi, se riferiti agli oggetti o concetti relativi a quel toponimo. Nella tabella 1 abbiamo classificato questo tipo di suffissi italiani come suffissi aggettivali. Visto che tutti gli aggettivi italiani in -o formano il genere femminile in $-a$, mentre gli aggettivi in $-a$ e in - $e$ hanno forma uguale al maschile e al

${ }^{2}$ Più raramente, al verbo detoponimico in -izovati corrisponde anche il nome verbale in -ovanje (amerikanizovanje). 
femminile, non è stato necessario includere le forme femminili nella tabella 1 , come abbiamo fatto nella tabella 2 per la lingua serba.

I suffissi italiani riscontrati ${ }^{3}$ sono i seguenti:

\begin{tabular}{|l|l|l|}
\hline \multicolumn{1}{|c|}{ nominali } & \multicolumn{1}{|c|}{ aggettivali } & verbali \\
\hline -ista & -(i)ota, -oto & -are \\
-istica & -(i)tano & -eggiare \\
-ismo & -acco & -izzare \\
-ità & -ano (-iano) & -ificare \\
-zione & -ardo & \\
& -asco & \\
& -ate & \\
& -atto & \\
& -eggiante & \\
& -eno & \\
& -ense & \\
& -eo & \\
& -esco & \\
& -ese & \\
& -iale & \\
& -ico (-itico) & \\
& -igiano & \\
& -ino & \\
& -istico & \\
& -ita & \\
& -occo & \\
& -olo & \\
& -otto & \\
& -Ø (suffisso zero) & \\
\hline
\end{tabular}

Tabella 1: Suffissi detoponimici italiani

Abbiamo registrato 5 suffissi nominali, 24 suffissi aggettivali e 4 suffissi verbali. Si può osservare che i suffissi aggettivali sono i più numerosi, e che ci sono solo 5 suffissi nominali, ma va ricordato che, a seconda del contesto, quasi tutti i suffissi etnici potrebbero rientrare anche nella categoria dei

${ }^{3}$ I suffissi etnici qui riportati sono elencati secondo la versione più concisa di Dardano (2009: 109-110). Grossmann e Rainer (2004: 405-408) ne elencano, invece, ben 34, secondo la statistica e gli studi di Crocco Galeas (1991), che non includono gli etnici extraitaliani. I suffissi etnici omessi dalla tabella 1 vengono usati raramente e sono relativi a pochi toponimi, in alcuni casi a un solo esempio: -ata, -aro (-are, -ere, -ero), -aiolo (-arolo), -ito, -ingo (-engo), -ente, -one, -erno, -oo, -ello, -inco, -occo, -ore, -uso, -acino, -acolo, -ertino (-urtino), suffissi greci: '-io, -iaco, -aico. 
suffissi nominali. Tra i suffissi verbali italiani, le forme detoponimiche in -are (ad es. italianare) sono assai rare e oggi soppiantate dai verbi in -izzare, per cui questo suffisso ${ }^{4}$ non viene preso in considerazione nella tabella 5 .

La situazione diventa più complessa nella lingua serba, per via dei differenti approcci alla suffissazione proposti da diverse opere analizzate.

\begin{tabular}{|l|l|l|}
\hline \multicolumn{1}{|c|}{ nominali } & \multicolumn{1}{|c|}{ aggettivali } & \multicolumn{1}{c|}{ verbali } \\
\hline -ac / -ka, -kinja, -ica & 1. -anski, -inski & -čiti \\
-(j)anac / -(j)anka, -ijanac & 2. -ački & -ificirati \\
-(j)ak / -(j)anka & 3. -ski, -čki, -ški, -ki & -iti \\
-ak / -kinja & 4. -ejski & -izovati, -izirati \\
-an / -anka & 5. -istički & -ovati \\
-anin (-čanin, -janin, & \\
-ljanin) / -anka (-čanka, & \\
-janka, -ljanka) & \\
-ejac / -ejka & \\
-ez / -eskinja & \\
-elj / -eljka & \\
-ica & \\
-in / -ka & \\
-inac / -inka & \\
-inja, -kinja & \\
-ist(a) & \\
-istika & \\
-izam & \\
-je & \\
-ka (da m. -ac) & \\
-lija / -lijka & \\
-ština & \\
-uša & \\
$\varnothing /$-ka, -ica, -(k)inja & \\
\hline
\end{tabular}

Tabella 2: Suffissi detoponimici serbi

La lista dei sufissi nominali varia da soli 4 maschili e 4 femminili (Klajn 2007), a 9 m. e 8 f. in Piper \& Klajn (2014). Gli studi sulla formazione delle parole presentano, naturalmente, un panorama più dettagliato di questo

${ }^{4}$ A essere precisi, il morfema -are non è un suffisso, ma la desinenza dell'infinito per i verbi della prima coniugazione, mentre i suffissi "propri” sarebbero -izz-, -egg-, -ific- o il suffisso zero (Grossmann e Rainer 2004: 450); comunque, per semplificare questa analisi, tratteremo anche la desinenza -are come parte del suffisso verbale. Lo stesso vale per le forme dell'infinito nella lingua serba. 
campo lessicale: così Klajn (2003) elenca 15 suffissi maschili, 6 femminili e uno che forma nomi di genere neutro $(-j e)$.

Tra i suffissi aggettivali si riscontrano meno differenze: le grammatiche serbe riportano di solito 3 suffissi aggettivali (-ski, -ški, -čki), ma in alcune, come quella normativa di Piper \& Klajn $(2014)$ e in Klajn $(2003,2007)$ viene citato solo il suffisso -ski, mentre tutte le altre forme sono considerate le sue varianti, allomorfe o composte (-ki, -čki, -ški; -anski, -inski, -ački, -ejski, -istički).

Infine, i suffissi verbali sono presentati soltanto in Klajn (2003), che riporta i 5 presenti nella tabella 2 .

Considerate le differenze di terminologia e d'uso, la tabella 2 riporta i suffissi largamente presenti nelle opere analizzate (sebbene solo alcuni tra i suffissi elencati siano produttivi), mentre vengono omesse alcune varianti di etnici regionali, limitate a pochi esempi meno conosciuti. Ciò risulta in 15 suffissi nominali maschili, cui corrispondono 13 suffissi femminili (incluse le varianti) e 1 neutro, 7 suffissi aggettivali e 5 suffissi verbali. Le differenze nel numero di suffissi riscontrati sono dovute anche al concetto diverso di variante; cioè, alcune grammatiche classificano molti esempi come varianti di un solo suffisso (ad es. -ano e-iano, o-ski e-čki), mentre altre li trattano come suffissi autonomi.

Possiamo constatare che nella lingua serba esistono vari suffissi allomorfi (con più varianti). La variante del suffisso che verrà aggiunta per formare detoponimici dipende dall'ultima consonante del nome di base, cioè del tipo di alternanza fonetica che questa consonante, o anche vocale, eventualmente richiede; ne elencheremo gli esempi in seguito.

\subsection{Suffissi nominali}

Tra i suffissi nominali nelle lingue confrontate, ad esclusione degli etnici, possiamo individuare molti tratti paralleli, sia morfologici che semantici.

\begin{tabular}{|l|l|}
\hline \multicolumn{1}{|c|}{ Suffissi nominali italiani } & \multicolumn{1}{|c|}{ Suffissi nominali serbi (etnici esclusi) } \\
\hline -ista: italianista, arabista, russista, serbista & $\begin{array}{l}\text {-ist(a): italijanist(a), arabist(a), rusist(a), } \\
\text { srbist(a) } \\
\text {-kinja /(raro -ica) }\end{array}$ \\
\hline $\begin{array}{l}\text {-istica: italianistica, croatistica, russistica, } \\
\text { serbistica }\end{array}$ & $\begin{array}{l}\text {-istika: italijanistika, kroatistika, } \\
\text { rusistika, srbistika }\end{array}$ \\
\hline $\begin{array}{l}\text {-ismo: grecismo, anglicismo, ispanismo, } \\
\text { turchismo }\end{array}$ & $\begin{array}{l}\text {-izam: grecizam, anglicizam, } \\
\text { hispanizam, turcizam }\end{array}$ \\
\hline -ità: italicità, sicilianità, milanesità & $\begin{array}{l}\text {-ština: engleština, francuština, } \\
\text { italijanština }\end{array}$ \\
\hline
\end{tabular}




\begin{tabular}{|l|l|}
\hline $\begin{array}{l}\text {-zione: americanizzazione, arabizzazione, } \\
\text { germanizzazione, russificazione }\end{array}$ & $\begin{array}{l}\text {-cija: amerikanizacija, arabizacija, } \\
\text { germanizacija, rusifikacija }\end{array}$ \\
\hline & $\begin{array}{l}\text {-ina }(+ \text { po-, deriv. parasintetica): } \\
\text { Sava }>\text { Posavina, Drava }>\text { Podravina }\end{array}$ \\
\hline & $\begin{array}{l}\text {-je }(+ \text { po-, } \text { za-, deriv. parasintetica): } \\
\text { Dunav }>\text { Podunavlje, Sava }>\text { Posavlje, } \\
\text { Tisa }>\text { Potisje }\end{array}$ \\
\hline
\end{tabular}

Tabella 3: Suffissi nominali

I suffissi italiani detoponimici -ista, -istica, -ismo, -zione, e in misura più ridotta anche -ità, trovano i propri equivalenti nei suffissi serbi -ista, -istika, -izam, -cija e, parzialmente, -ština. Questi suffissi producono sostantivi detoponimici, soprattutto dalle basi aggettivali e nominali (che possono includere anche i nomi delle lingue), tranne il suffisso -zione/-cija, che è deverbale ed è associato ai verbi in -izzare.

Tra quelli elencati, il solo a riferirsi a persone è il suffisso -ista, che in italiano ha la stessa forma al singolare per il genere maschile e femminile, mentre in serbo per il genere femminile viene usato il suffisso-kinja o più raramente -ica - it. italianista, m. f. - sr. italijanist(a), m., italijanstkinjal italijanistica $\mathrm{f}$.

Il suffisso -ista è tra i più produttivi suffissi italiani in generale, ma nel caso dei detoponimici si riferisce alla professione di studiosi di cultura, lingua o letteratura dei popoli, il cui nome, o il nome della cui lingua, rappresenta la base ${ }^{5}$ (Grossmann e Rainer 2004: 14; Klajn 2003: 244). In questo modo, si associa ai suffissi -istica/-istika che formano i nomi femminili per i rispettivi ambiti di studio - it. italianistica, sr. - italijanistika (Grossmann e Rainer 2004: 248), nonché al suffisso -ismo/-izam che nella sua accezione detoponimica forma i termini linguistici per i forestierismi originari dalle lingue nella base (italianismo - italijanizam). Va notato che la radice per tutti i suffissi elencati è spesso grecizzante o latineggiante, soprattutto notevole nella lingua serba che ha forme differenti di toponimi di base (Klajn 2003: 244): it. inglese > anglista, anglistica, angli(ci)smo, sr. engleski>anglista/-kinja, anglistika, anglicizam, hrvatski> kroatista, kroatistika, kroatizam, crnogorski>montenegrista, montenegristika (ma non per srpski>srbista, srbistika), ecc. In accezioni più astratte, -ismo/-izam può

${ }^{5}$ Negli esempi come milanista o romanista, che indicano tifosi delle squadre del Milan o della Roma, la radice non è propriamente un toponimo, cioè il nome della città, ma quello della squadra. Comunque, romanista può avere l'accezione di studioso di Roma o delle lingue e letterature romanze. 
indicare il comportamento o un tratto tipico del toponimo o etnico di base, oppure l'ammirazione per tutto quello che ne proviene (americano>americanismo, Balkan>balkanizam).

Il suffisso -ità genera nomi astratti femminili che, se la radice è un toponimo o un etnico, indicano una qualità intrinseca alla cultura o alla personalità degli abitanti del toponimo in questione: italianità, sicilianità, grecità, slavità, lombardità ecc. Vi corrisponde in parte il suffisso serbo -ština (Klajn 2003: 210), il quale, però, se riferito alle qualità astratte introduce per lo più una sfumatura negativa o perfino spregiativa (amerikanština, evropejština, jevrejština, francuština).

I nomi verbali in -zione e -cija, che esprimono l'azione prodotta dai verbi in -izzare (-izirati-izovati), o in casi piuttosto rari, quelli in -ificare (-ificirati), sono praticamente equivalenti nell'italiano e nel serbo.

Invece, solo nella lingua serba sono riscontrabili i suffissi detoponimici che formano altri toponimi mediante la derivazione prefissale-suffissale, cioè parasintetica, con l'aggiunta del prefisso po- e dei suffissi -ina e -je. Questi derivati parasintetici indicano più spesso regioni attraversate da fiumi, $i$ cui nomi di solito sono alla base, talvolta con alcune alternanze fonetiche: Morava $>$ Pomoravlje, Sava $>$ Posavina. Il suffisso -ina crea toponimi femminili, mentre -je crea nomi di genere neutro.

\subsection{Suffissi aggettivali}

Dato che i suffissi italiani che formano aggettivi etnici sono ovviamente molto più numerosi, qui ne abbiamo individuati soltanto i più produttivi, per poterli paragonare ai suffissi detoponimici aggettivali nella lingua serba.

\begin{tabular}{|l|l|}
\hline \multicolumn{1}{|c|}{ Suffissi aggettivali italiani più produttivi } & \multicolumn{1}{|c|}{ Suffissi aggettivali serbi } \\
\hline $\begin{array}{l}\text {-ese (-ense): milanese, francese, inglese, } \\
\text { monacense, parmense, panamense }\end{array}$ & $\begin{array}{l}\text {-ski (-čki, -ški, -ki): italijanski, američki, } \\
\text { njujorški, bečki }\end{array}$ \\
\hline -(i)ano: americano, italiano, peruviano & -anski, -inski: venecijanski, vizantinski \\
\hline -ino: parigino, montenegrino, spalatino & -ački: slovenački, zagrebački, kruševački \\
\hline -ico: britannico, balcanico, germanico & -ejski: korejski, evropejski \\
\hline -istico: italianistico, slavistico, anglistico & -istički: rusistički, slavistički, anglistički \\
\hline
\end{tabular}

\section{Tabella 4: Suffissi aggettivali}

Osserviamo alcune differenze interessanti in queste due colonne, dovute alle differenze tra l'italiano e il serbo dal punto di vista della flessione:

- mentre i suffissi italiani aggettivali sono ovviamente più numerosi, tra i suffissi aggettivali serbi si riscontrano molte varietà allomorfe 
degli stessi suffissi, e così ad esempio, a seconda della terminazione del tema, il suffisso -ski può diventare -ški, -čki o semplicemente - $k i$, per via delle diverse alternanze fonetiche (Klajn 2007: 180). Sono inoltre assai comuni suffissi composti dal morfema -ski e un altro morfema che gli precede, come nei casi di -anski, -inski ecc;

- nella tabella sono riportati soltanto gli esempi di genere maschile, per mancanza di spazio. Gli aggettivi italiani maschili in -o hanno la forma per il genere femminle in $-a$, a differenza degli aggettivi in $-e$ che hanno la stessa forma per entrambi i generi. La lingua serba conosce invece tre generi: maschile, femminile e neutro, e mentre gli aggettivi maschili al nominativo finiscono in $-i$, gli aggettivi femminili avranno la desinenza in $-a$, e quelli neutri in -o: venecijanski karneval, italijanska zastava, njujorško pozorište;

- $\quad$ i suffissi italiani -ese (-ense), -(i)ano e -ino possono essere sia nominali che aggettivali, quando formano gli etnici. Ciò vale anche per il suffisso -ico in misura più limitata. Invece il suffisso italiano -istico, come tutti i suffissi aggettivali nella colonna a destra, è esclusivamente aggettivale.

\subsection{Suffissi verbali}

I suffissi verbali sono pochi, ma produttivi in entrambe le lingue analizzate. Tutti i suffissi produttivi italiani appartengono alla la coniugazione (o classe flessiva).

\begin{tabular}{|c|c|}
\hline Suffissi verbali italiani & Suffissi verbali serbi \\
\hline & Derivazione parasintetica (+ prefisso po-) \\
\hline & -čiti: pon(j)emčiti, poturčiti \\
\hline & -iti: posrbiti, poengleziti, potalijaniti \\
\hline \multirow{2}{*}{$\begin{array}{l}\text {-eggiare: latineggiare } \\
\text { (-eggiante: latineggiante) } \\
\text {-izzare: europeizzare, italianizzare, } \\
\text { germanizzare, sanremizzare } \\
\text { (-izzante: grecizzante) } \\
\text {-ificare: russificare }\end{array}$} & Suffissazione \\
\hline & $\begin{array}{l}\text {-izovati, -izirati: evropeizovati, italijanizovati, } \\
\text { germanizovati, latinizirati, kroatizirati } \\
\text {-ovati: srbovati, hrvatovati (intr.) } \\
\text {-ificirati: rusificirati }\end{array}$ \\
\hline
\end{tabular}

Tabella 5: Suffissi verbali

La differenza subito evidente dalla tabella 5 è che nella lingua serba è talvolta presente anche la derivazione parasintetica (prefissale-suffissale), non prevista però nell'italiano. Nel caso dei suffissi verbali serbi, il suffisso -iti spesso richiede anche un prefisso per formare il verbo transitivo, di 
solito il prefisso po-: pon(j)emčiti, posrbiti, potalijaniti, il che corrisponde al suffisso italiano oggi più produttivo, -izzare: germanizzare, serbizzare, italianizzare. Anche il suffisso serbo della stessa origine, -izovati, con la varietà meno comune -izirati, è oggi molto più produttivo dei precedenti. Soltanto il suffisso -ovati forma verbi intransitivi.

Bisogna comunque fare una distinzione tra i suffissi verbali italiani, perché - eggiare, a differenza di -izzare, forma di solito verbi intransitivi, che spesso comportano una connotazione negativa e raramente sono detoponimici (in effetti, abbiamo riscontrato solo un esempio nel verbo latineggiare - "attenersi all'uso latino, o parlare o scrivere usando espressioni latine", questo è anche l'unico esempio del verbo italiano che non sia transitivo). Da notare anche le forme del participio presente in -eggiante, che appaiono anche nei verbi in -izzare come -izzante, come aggettivi col significato di imitazione o somiglianza (latineggiante, grecizzante).

I verbi in -izzare e -izovati-izirati, come pure alcuni sostantivi negli esempi della tabella 3, hanno frequentemente la radice latineggiante o grecizzante: croatizzare, anglicizzare, germanizovati, helenizovati.

\section{ETNICI}

I suffissi che formano gli etnici italiani sono, in generale, più numerosi dei suffissi etnici nel serbo, ma in entrambe le lingue confrontate i suffissi etnici sono più numerosi di quelli riportati nelle tabelle. Limitandoci ai suffissi più citati nella letteratura (soprattutto Dardano e Grossman e Rainer), abbiamo omesso quelli che compaiono in uno o due esempi regionali o dialettali, e abbiamo incluso i suffissi più rari solo se vengono menzionati più volte nelle diverse opere analizzate.

\begin{tabular}{|l|l|}
\hline \multicolumn{1}{|c|}{$\begin{array}{c}\text { Suffissi etnici italiani, } \\
\text { nominali e aggettivali }\end{array}$} & \multicolumn{1}{c|}{ Esempi } \\
\hline -(i)ota, -oto & cairota, cipriota, tokiota \\
\hline -(i)tano & napolitano, palermitano, anconetano \\
\hline -acco & polacco \\
\hline -ano (-iano) & italiano, africano, bostoniano \\
\hline -ardo & savoiardo, nizzardo \\
\hline -asco & comasco, bergamasco, monegasco \\
\hline -ate & ravennate, assisiate, urbinate \\
\hline -atto & bellunatto $(<$ Belluno), caprolatto $(<$ Caprarola $)$ \\
\hline -eno & cileno, iracheno, madrileno \\
\hline
\end{tabular}




\begin{tabular}{|l|l|}
\hline -ense & panamense, statunitense, ostiense \\
\hline -eo & europeo, raguseo \\
\hline -esco & pantesco ( $>$ Pantelleria) \\
\hline -ese & bolognese, irlandese, piemontese \\
\hline -iale & laziale, provenzale, badiale $(<$ Badia Tedalda $)$ \\
\hline -ico (-tico) & dolomitico, libico, asiatico \\
\hline -igiano & parmigiano, trevigiano, marchigiano \\
\hline -ino & fiorentino, reggino, montenegrino \\
\hline -ita & moscovita, vietnamita, istanbulita \\
\hline -occo & bustocco $(<$ Busto Arsizio $)$ \\
\hline -olo & spagnolo, romagnolo, brianzolo $(>$ Brianza) \\
\hline -otto & badiotto, chioggiotto, rovigotto \\
\hline -Ø (suffisso zero) & svizzero, toscano, ceco \\
\hline
\end{tabular}

Tabella 6: Suffissi etnici italiani

Nella lingua serba, come già notato nella sezione $1, i$ criteri per trattare un suffisso come autonomo o meno differiscono notevolmente in opere diverse, e anche qui l'elenco riportato è ridotto ${ }^{6}$.

\begin{tabular}{|c|c|}
\hline Suffissi etnici nominali serbi & Esempi \\
\hline -ac/-ka, -kinja, -ica & - Japanac/Japanka, Irac/Irkinja, Balkanac/Balkanka \\
\hline -(j)anac/(j)anka & - Amerikanac/Amerikanka, Sicilijanac/Sicilijanka \\
\hline $\begin{array}{l}\text {-(j)ak / -(j)anka, -kinja } \\
\text {-an/-anka } \\
\text {-anin (-čanin, -janin, -ljanin), / -anka } \\
\text { (-čanka, -janka, -ljanka) }\end{array}$ & $\begin{array}{l}\text { - Poljak/Poljakinja, Tuzlak/Tuzlanka } \\
\text { - Italijan/Italijanka } \\
\text { - Kotoranin/Kotoranka, Zagrepčanin/Zagrepčanka, } \\
\text { Rimljanin/Rimljanka }\end{array}$ \\
\hline $\begin{array}{l}\text {-ejac/-ejka } \\
\text {-ez/-eskinja } \\
\text {-in/-ka, -kinja, -inka } \\
\text {-inac/-inka } \\
\text {-lija/-lijka } \\
\varnothing /-k a,- \text {-ica, -(k)inja }\end{array}$ & $\begin{array}{l}\text { - Evropejac/Evropejka } \\
\text { - Kinez/Kineskinja, Englez/Engleskinja } \\
\text { - Jevrejin/Jevrejka, Srbin/Srpkinja, Gruzin/Gruzinka } \\
\text { - Dalmatinac/Dalmatinka, Firentinac/Firentinka } \\
\text { - Bečlija/Bečlijka, Nišlija/Nišlijka } \\
\text { - Brazil/Brazilka, Mađar/Mađarica, Čeh/Čehinja }\end{array}$ \\
\hline
\end{tabular}

Tabella 7: Suffissi etnici serbi, con la mozione femminile

Anche con queste liste ridotte, si osserva che il numero dei suffissi etnici italiani (22) è doppio rispetto ai suffissi etnici serbi (11, senza contare i suffissi di mozione femminile). Comunque, il numero totale dei suffissi etnici produttivi nelle due lingue confrontate non varia molto: in italiano

${ }^{6}$ Sono omesse le forme rare, limitate a uno o due esempi (-elj in Bokelj, -enac in Nazare$n a c,-i \check{s} n i k$ in Krajišnik ecc.), con l'eccezione di -ejac per la frequenza del termine Evropejac. 
i più produttivi sono i suffissi -ese (-ense), -ino e -(i)ano (Dardano 2009: 110), mentre in serbo lo sono soprattutto -ac, -anin e -anac (Klajn 2003: 52, 58; Piper \& Klajn 2014: 223).

Come già notato, la maggior parte degli etnici italiani, con alcune eccezioni, può essere usata sia in funzione nominale che aggettivale, mentre gli etnici serbi mantengono forme separate per nomi e aggettivi: Portogallo>portoghese, lingua portoghese; Portugal(ija)>Portugalac/Portugalka, portugalski jezik. Va notata anche la grafia differente, nel senso che gli etnici in italiano di solito non vengono scritti con la maiuscola, se non si tratta dei popoli antichi, mentre la maiuscola per gli etnici nominali serbi è obbligatoria, e per gli etnici aggettivali si usano invece sempre le minuscole.

Mentre nei deonomastici etnici italiani nominali e aggettivali, come -ano o -esco, il genere femminile viene derivato semplicemente cambiando la desinenza -o in -a (italiano>italiana), oppure la desinenza in - $e$ o - a come -ese o -ista include entrambi i generi al singolare (il milanese/ la milanese, il milanista/la milanista), nella lingua serba questo avviene soltanto negli aggettivi, che conoscono anche il genere neutro (francuski) francuska/francusko), mentre per la derivazione dei nomi femminili esistono appositi suffissi, soprattutto quando si tratta di etnici (Italijan>Italijanka, Grk $>$ Grkinja, Mađar $>$ Mađarica, ecc.). Dalla tabella 7 è ovvio che i suffissi di mozione femminile sono molto più numerosi nel serbo.

A proposito dell'influenza di altre lingue, tra i suffissi italiani etnici ci sono più suffissi stranieri che tra quelli serbi (Grossmann e Rainer 2004: 406-408; Klajn 2003: 223, 227, 241). Gli influssi forestieri sono individuabili nei suffissi -oto, -(i)ota, -ita, -iaco, -aico (greci), -eno (dallo spagnolo -eño, per il quale esiste anche la variante italiana rara -egno), o -ardo (francese). Invece nel serbo riscontriamo soltanto -ez (dall'italiano -ese: Kinez, Milanez, Pijemontez) e talvolta -inac (probabilmente latino7: Firentinac, Vizantinac) o molto raramente -alac (francese ${ }^{8}$ : Provansalac).

Una caratteristica degli etnici italiani, che non è presente in quelli serbi, è la loro frequente tendenza latineggiante, quando come base di derivazione si usa un antico toponimo latino. Ne riscontriamo esempi anche tra i suffissi più produttivi: Chieti $>$ teatino, Ivrea $>$ eporediese, Montepulciano $>$ poliziano. Questa tendenza negli etnici italiani si spiega con "l'intento di conferire alla propria città o paese una dignità superiore, mettendo in risalto la sua antichità" (Grossmann e Rainer 2004: 402).

Entrambe le lingue confrontate conoscono il cosiddetto suffisso zero, specialmente usato nella formazione di etnici: Grecia $>$ greco, Grčka>Grk; Russia>russo, Rusija>Rus; Repubblica Ceca>ceco, Češka>Čeh, ecc.

\footnotetext{
${ }^{7}$ Cfr. Klajn (2003: 241).

${ }^{8}$ V. Klajn \& Šipka (2008).
} 
Naturalmente sono più frequenti i casi in cui l'uso del suffisso zero in italiano non corrisponde al suffisso zero in serbo, ma invece viene usato un altro suffisso: Slovenia $>$ sloveno, Slovenija $>$ Slovenac; Svizzera $>$ svizzero, Švajcarska $>$ Švajcarac ecc.

\section{CONCLUSIONE}

Nella ricerca presentata abbiamo analizzato il campo onomastico dei detoponimici suffissati nelle lingue italiana e serba.

I suffissi detoponimici nelle lingue confrontate sono nominali, aggettivali e verbali, e appartengono per la maggior parte a suffissi denominali, visto che la base di derivazione sono toponimi. Comunque, nel caso dell'accumulazione dei suffissi, la base può essere anche un aggettivo etnico o un verbo in -izzare/-izovati.

In questo campo, i derivati etnici (nomi e aggettivi) sono di gran lunga i più numerosi; sebbene la ricerca non includa soltanto gli etnici, risulta chiaro che i suffissi che formano gli altri tipi di detoponimici sono piuttosto scarsi, anche se produttivi.

É stato necessario ridurre l'elenco dei suffissi etnici riscontrati in entrambe le lingue analizzate. L'abbiamo fatto tenendo conto soprattutto dei suffissi elencati in più opere diverse, omettendo quelli di carattere regionale o dialettale. Anche con questa riduzione, nel numero totale dei suffissi elencati, si nota la sproporzione nella categoria morfologica contenente gli etnici (aggettivi nell'italiano, nomi nel serbo) rispetto alle altre due, limitate a 4-5 suffissi. La difficoltà nell'analisi del materiale onomastico serbo è consistita soprattutto negli approcci linguistici diversi. È naturale che gli studi dedicati alla formazione delle parole (Klajn 2003) siano più esaurienti delle grammatiche generali.

Bisogna inoltre ribadire che, mentre la toponomastica italiana dispone di opere voluminose quale il Dizionario degli etnici e dei toponimi italiani (DETI), che registra più di 8000 toponimi ed etnici, la lingua serba non possiede una fonte simile, ma solo studi sparsi sui toponimi locali di zone geografiche limitate, oppure quelli che si concentrano su un aspetto particolare?.

Le equivalenze più evidenti tra l'italiano e il serbo si riscontrano nei suffissi di origine greca -ista, -ismo, -istico, -istica, -izzare (-ist(a), -izam, -istički, -istika, -izirati/-izovati) i quali, insieme all" "internazionale" e latineggiante -zione (-cija), sono anche i più produttivi tra i suffissi "non etnici". Possiamo concludere che si tratta di componenti "internazionali",

${ }^{9}$ Jelić (2015); Duran (2017). Sono inoltre stati pubblicati diversi articoli sull'argomento (Fekete 1982, Georgijević 1981, Pešikan 1958/1959 ecc.). 
che non si limitano soltanto alle lingue analizzate in questa sede. Visto che si tratta di due tipi di lingue, così diverse come lo sono le lingue slave e quelle romanze, è da aspettarsi che $i$ tratti in cui più si assomigliano appartengano a un ambito comune più esteso.

Si nota un influsso italiano nel suffisso etnico $-e z$, mentre non sono riscontrate le influenze serbe sui suffissi italiani. Tra gli etnici, i suffissi più produttivi sono gli italiani -ese (-ense), -ino e -(i)ano, e nel serbo -ac, -anin e-anac. Inoltre, mentre l'italiano per il genere femminile al singolare conosce soltanto le desinenze $-a$ ed $-e$, il serbo annovera ben 8 suffissi solo per la mozione femminile (e con un approccio più largo se ne possono interpretare anche 13).

Benché la prima associazione alla derivazione detoponimica siano gli etnici, la nostra ricerca ha rivelato che un numero ridotto di suffissi detoponimici può avere altri significati (professione, ambito di studio, qualità astratta, parola straniera, azione legata all'etnico di base ecc.). Questo campo di studi merita delle ricerche ulteriori, soprattutto perché gli argomenti onomastici nella lingua serba non sono stati ancora approfonditi fino in fondo.

\section{BIBLIOGRAFIA}

Crocco Galeas, G. (1991). Gli etnici italiani. Studio di morfologia naturale. Padova: Unipress.

Dardano, M. (2009). Costruire parole: la morfologia derivativa dell 'italiano. Bologna: Il Mulino.

Dizionario degli etnicie dei toponimi italiani (DETI). (2017). Bologna: Patron. Duran, T. (2017). Toponimi turskog porekla u Srbiji (dottorato di ricerca). Beograd: Filološki fakultet, Univerzitet u Beogradu.

Fekete, E. (1982). Nešto o obradi ktetika u našim savremenim rečnicima. In D. Ćupić (a cura di), Leksikografija i leksikologija: zbornik referata (pp. 317-324). Beograd: SANU.

Georgijević, S. (1981). O problemima patronima, etnika i ktetika u srpskohrvatskom jeziku. Onomatološki prilozi, II, 123-142.

Grossmann, M. e Rainer, F. (2004). La formazione delle parole in italiano. Tübingen: Max Niemeyer Verlag.

Jelić, M. (2015). Etnici i ktetici u Vojvodini. Sombor: Univerzitet u Novom Sadu / Pedagoški fakultet u Somboru.

Klajn, I. (2003). Tvorba reči u savremenom srpskom jeziku II: sufiksacija i konverzija. Beograd: Zavod za udžbenike i nastavna sredstva - Institut za srpski jezik SANU; Novi Sad: Matica srpska.

Klajn, I. (2007). Grammatica della lingua serba. Belgrado: Zavod za udžbenike i nastavna sredstva. 
Klajn, I. \& Šipka, M. (2008). Veliki rečnik stranih reči i izraza. Novi Sad: Prometej.

Neologismi: parole nuove dai giornali (2009). Roma: Istituto Treccani.

Pešikan, M. (1958/1959). O građenju imena stanovnika u odnosu na imena zemalja i mesta. Naš jezik IX, 5-6, 196-205.

Piper, P. \& Klajn, I. (2014). Normativna gramatika srpskog jezika. Novi Sad: Matica srpska.

\section{DETOPONYMICAL SUFFIXES IN ITALIAN AND SERBIAN}

\section{Summary}

The paper presents the process of deriving deonomastic terms from toponyms by means of suffixes, through the research which compares Italian detoponymical suffixes with those in Serbian language. Using the contrastive approach, the paper analyzes morphological and semantic differences and similar features, with morphological classification of derived words as a starting point. The results show that suffixed terms derived from toponyms represent an onomastic field that does not include only demonyms (romano, belgradese, parigino), but also other derived terms that are productive in both compared languages (italianista, arabismo, slavistico). However, demonyms and their derivations remain the most numerous part and can become basic words for other detoponymical suffixes (greco>grecizzare>grecizzazione). The number of productive suffixes in the compared languages is limited, and most reciprocally similar features are discovered in "international" suffixes, of Greek or Latin origin.

Keywords: detoponymical suffixes, toponyms, suffixation, demonyms, Italian, Serbian. 